\title{
Assessment of Laparoscopic Intraoperative Cholangiogram in Controlling Postoperative Outcomes and Its Implication on the Quality of Life
}

\author{
Mohamed A. Elkerkary ${ }^{*}$, Ahmad Hasssan, and Hamdy Shaban \\ Department of Surgery, Faculty of Medicine, Suez Canal University
}

\begin{abstract}
Background: Intraoperative cholangiography (IOC) is one of the surgical advances that aim to reduce the risk of bile duct injuries. Aim: To assess the role of $I O C$ in controlling the postoperative outcomes and its implication on patient quality of life. Patients and Methods: This prospective randomized study conducted at the Department of Surgery, Faculty of Medicine, Suez Canal University from May 2017 to February 2019 on 56 patients with symptomatic gall stones who met our inclusion criteria. The exclusion criteria were having former upper abdominal surgery, chronic liver disease, impaired kidney function, bleeding tendency, and Common bile duct (CBD) stones indicated for preoperative ERCP. Our protocol was approved by the institutional review board at Suez Canal University. Results: Our sample comprised of 56 patients. The mean age was $42 \pm 10$ years, and most patients were females (68\%). Most patients had no interventions like CBD explorations ( $82 \%$ ). The success rate was $96 \%$. Two patients had failed CBD exploration and referred to postoperative Endoscopic Retrograde Cholangiopancreatography (ERCP). Of notice, there were no reported cases of bile duct injury, and the mortality rate was $0 \%$. Conclusion: IOC during laparoscopic cholecystectomy (LC) is associated with a low morbidity rate and no mortalities. This confirms the effectiveness of IOC in reducing the postoperative complications of LC which improve patent's quality of life.
\end{abstract}

Keywords: Intraoperative Cholangiography; Laparoscopic Cholecystectomy; Quality of Life

\section{Introduction}

Despite the known benefits of Laparoscopic cholecystectomy (LC) as a gold standard procedure for gallstone disease, there are still some concerns about the most severe complication of this procedure, injury of the bile duct (BDI) $)^{(1,2)}$. Complex BDIs and late complications may lead to end-stage liver disease and associated with an increased incidence of mortality rate (up to $7 \%$ ). However, with the great advances in this surgical procedure and with the rising of the learning curve of the surgeons, the incidence of BDIs showed a significant reduction compared with earlier periods(3,4). Intraoperative cholangiography (IOC) is one of these advances that aim to reduce the risk of BDIs $(5,6)$. Different solutions are available when the 
BDI is diagnosed during surgery or in those patients who have received multiple prior therapies, and a multidisciplinary approach is essential $(7,8)$. However, the resolution of a diagnosed BDI after surgery is purely surgical. Moreover, IOC must be performed to give a surgical "road map" when the anatomical features of the common bile duct (CBD) are unclear during the procedure ${ }^{(9)}$. Some studies reported a significant reduction in the mortality rates, morbidity, cardiac events, bleeding, reintervention, and postoperative biliary complications with early detection of BDIs detection during $\operatorname{IOC}^{(10,11)}$. LC with $I O C$ is observed to be associated with a lower risk of readmission related to the first operation $^{(12)}$. On the other hand, some studies reported some limitations for the use of $\operatorname{IOC}^{(1,13)}$. Therefore, they suggested that IOC should be used only in selected cases. However, in some cases with difficult intraoperative anatomical findings, $I O C$ is the treatment of choice ${ }^{(14)}$. Therefore, in this study, we aimed to assess the role of $I O C$ in controlling the postoperative outcomes and its implication on the quality of life.

\section{Patients and Methods}

\section{Study design}

The current study was performed as a prospective randomized study at the Department of Surgery, Faculty of Medicine, Suez Canal University from May 2017 to February 2019.

\section{Patients}

Adult patients with good general condition were enrolled if they were diagnosed with symptomatic gall stones. The exclusion criteria were having former upper abdominal surgery, contraindications to the contrast material, chronic liver disease, impaired kidney function, bleeding tendency, and CBD stones indicated for preoperative ERCP. Our protocol was ap- proved by the institutional review board at Suez Canal University and all participants signed an informed written consent before the procedure.

\section{Operative Interventions}

Following general anesthesia, LC with cholangiography was performed using the standard four-cannula technique. We applied traction on the gallbladder fundus in a cephalic direction with a grasper placed through the midclavicular port. Then, we performed intraoperative cholangiography using either a cholangiogram/a ureteric catheter or catheter $(5 \mathrm{~F})$ or a specialized grasper with the cholangioneedle catheter. Using the first catheter, it was guided into the partially transacted proximal cystic duct (using a guide wire when necessary) and was secured in place in the cystic duct with a cholangioclamp. The second catheter was used to inject 5 to $15 \mathrm{ml}$ of contrast material to visualize the biliary ductal system. Then, the biliary duct was flushed with saline. The catheter was removed, two clips placed on the common duct end of the cystic duct and it was divided with scissors.

\section{Data collection}

We collected the following data: demographics, experienced symptoms, comorbid conditions, and current history of cholangitis or pancreatitis. Patients presenting with acute cholangitis or pancreatitis were allowed to settle first before the operation. In patients presenting with jaundice, abnormal LFTs or unresolved cholangitis, preoperative ERCP with or without sphincterotomy was performed. We further performed abdominal examination to assess organomegaly, positive Murphy's sign and any signs of cholangitis. The following preoperative investigations were performed: $C B C$, INR, liver, and renal function tests. Later, all participants underwent abdominal US imaging 
to detect dilated CBD, intra hepatic biliary radical dilatation.

\section{Discharge and follow-up}

Patient discharge was allowed after clinical improvement, defined as a reduction of abdominal pain and tenderness, normalization of laboratory values and neutral fluid balance. Following discharge, patients were followed up clinically (abdominal pain, guarding, masses) every 2 weeks for one month, then every month for 3 months and radiologically (abdominal US) if indicated. To detect late postoperative complications, we continued the follow up for six months.

\section{Outcome measures}

Safety was determined based on the rate of IOC-associated complications, morbidity using morbidity rate (incidence of all complications divided by the number of all patients) and postoperative morbidity index (each complication was given a score according to severity), mortality rate, conversion rate, and readmission rate.

\section{Statistical analysis}

For descriptive statistics, we used the mean \pm standard deviation, while for categorical parameters, we used the count (\%). To analyse the association between baseline data and outcomes, we used Chisquare or Fisher's Exact tests (for categorical) and $t$ test (for numerical data). All statistical analyses were performed using the SPSS (version 22 for Windows, IBM, Armonk, NY). A two-sided p-values <0.05 were considered statistically significant.

\section{Results}

\section{Baseline characteristics}

Our sample comprised 56 patients. The mean age was $42 \pm 10$ years, and most patients were females (68\%). Most patients were non-smokers (86\%) and only 21 pa- tients had comorbid chronic illnesses. About $91 \%$ of patients had elective LC, indicated for LC included acute calcular cholecystitis, chronic calcular cholecystitis with history of pancreatitis. Over three fourths of the patients had normal LFTs and total leukocytic count. In terms of preoperative US findings, nearly two thirds of patients had normal ultrasound while one third had dilated intrahepatic biliary duct IHBD (13\%) and CBD sludge (16\%). The baseline characteristics are illustrated in Table 1.

\section{Success and conversion rates:}

The majority of patients had no intervention (82\%). The success rate was $96 \%$. Two patients had failed CBD exploration and referred to postoperative ERCP. Another two cases were acceded for conservative management. (Flushing the CBD and clearance intraoperative). Three cases had flushing and milking of stone at the distal cystic duct. (Junction of cystic duct and (BD), while another three cases converted to open cholecystectomy. None of the patients had CBD exploration either transcystic or direct. There was barely any need to conversion to open cholecystectomy except in three cases because of finding of adhesions or fibrous bands, and impacted stones; Tables $2 \& 3$.

\section{Postoperative complications}

Over three fourths of patients stayed at hospital for one day after the surgery. Only nine percent had prolonged hospital stay; $7 \%$ for only three days, and $2 \%$ for four days. Approximately over three fourths of the patients stayed at hospital for 24-hours only while less stayed for more than a day. Postoperative complications encompassed intra-abdominal collection, wound infection, pancreatitis, bile leak, bleeding, and postoperative hernia. Of notice, there was no reported cases of bile duct injury, and mortality rate was $0 \%$; Table 4. 


\begin{tabular}{|l|c|c|}
\hline \multicolumn{3}{|c|}{ Table 1: Demographic characteristics of the patients } \\
\hline Parameters & $\mathrm{N}$ & $\%$ \\
\hline Age (years) mean \pm SD & $42 \pm 10$ \\
\hline Gender & & \\
Male & 18 & 32 \\
Female & 38 & 68 \\
\hline Residency & & \\
Urban & 29 & 52 \\
Rural & 27 & 48 \\
\hline Smoking & 8 & 14 \\
\hline Special habits “addict” & 1 & 2 \\
\hline Chronic diseases & & \\
Hypertension & 8 & 14 \\
DM & 7 & 12 \\
Chronic liver disease & 4 & 7 \\
IHD & 2 & 4 \\
\hline Indication of LC & & \\
Acute calcular & 5 & 9 \\
Chronic calcular cholecystitis & 46 & 82 \\
Chronic calcular cholecystitis with history of pancreatitis & 5 & 9 \\
\hline Type of surgery & & \\
Elective & 51 & 91 \\
Emergency & 5 & 9 \\
\hline Laboratory Parameters & & \\
High AST & 13 & 23 \\
High ALT & 13 & 23 \\
High ALP & 1 & 2 \\
High Total Bilirubin & 5 & 9 \\
High TLC & 5 & 9 \\
\hline AST: Aspartate Transaminase, ALT: Alanine Transaminase, ALP: Alkaline Phos- \\
phatase, TLC: total leukocytic count, DM: diabetes mellitus, IHD: ischemic heart \\
disease & & \\
\hline
\end{tabular}

Follow-up

Concerning three months of follow up, fewer percent complained of abdominal pain (5\%), cholangitis (2\%), abnormal LFTs (5\%), and only $5 \%$ of patients were readmitted to hospital after one month. By the second month of follow up, only $2 \%$ of patients had cholangitis and $2 \%$ had abnormal LFTs. After the third moth of follow up, none of the patient had abdominal pain, jaundice pancreatitis, cholangitis, abnormal LFTs, or was readmitted; Table 5 .

\section{Discussion}

Over the year, several strategies have been employed to minimize the incidence of $\mathrm{BDI}^{(16)}$, including use of intraoperative cholangiography (IOC), laparoscopic US, cholecystocholangiography and the critical view of safety ${ }^{(17)}$. However, the success of any of these techniques depends on accurate interpretation of normal biliary anatomy, anatomical variations and abnormal findings ${ }^{(18)}$. Here, we aimed to 
assess the efficacy of $I O C$ in reducing $L C$ postoperative complications. Our results showed that none of our cases had BDI, however there were few complications.

\begin{tabular}{|l|c|c|}
\hline Table 2: Interventions done during LC & N & $\%$ \\
\hline Intervention & 46 & 82 \\
\hline NAD & 2 & 4 \\
\hline Failed CBD exploration, post-Operative ERCP & 3 & 5 \\
\hline Flushing and milking of stone at the distal cystic duct & 3 & 5 \\
\hline Conversion to Open & 2 & 4 \\
\hline Conservative management & 0 & 0 \\
\hline Transcystic CBD exploration/ Direct CBD exploration & Nat \\
\hline NAD: no abnormality described, CBD: common bile duct, \\
ERCP: Endoscopic Retrograde Cholangiopancreatography \\
\hline
\end{tabular}

The mortality rate was $0 \%$. The most common complication was wound infection ( $\mathrm{N}=2$ ) followed by equal frequencies of intra-abdominal collection, pancreatitis, bile leak, bleeding, and postoperative hernia $(N=1)$. None of the cases in our study had BDI. This is in agreement with a study of 190 patients, where Khan et al. (19) found that the incidence of BDI among patients who underwent LC without $I O C$ is $1 \%$ in comparison to $0 \%$ among those who had IOC.

\begin{tabular}{|l|c|c|}
\hline Table 3: Conversion rate and causes of conversion \\
\hline Variables & $\mathrm{N}$ & $\%$ \\
\hline Conversion rate & 3 & 5 \\
\hline Cause of conversion & & \\
$\quad$ Adhesions/fibrous bands & 2 & 4 \\
$\quad$ Impacted stones & 1 & 2 \\
\hline
\end{tabular}

Similarly, Tornqvist et al. (20) reported a $1.5 \%$ incidence rate of $\mathrm{BDI}$ in $\mathrm{LC}$ without using IOC. IOC decreased this high incidence of BDI up to the half(21). Further, Buddingh et al. concluded that routine $\mathrm{IOC}$ reduced the rates of $\mathrm{BDI}^{(22)}$. Despite the $0 \% \mathrm{BDI}$ incidence, we had few cases that developed early and late postoperative complications. The most common complication was wound infection $(\mathrm{N}=2)$ followed by equal frequencies of intraabdominal collection, pancreatitis, bile leak, bleeding, and postoperative hernia $(\mathrm{N}=1)$. Similarly, Photi et al. found that wound infection was the most common postoperative complication, followed by bleeding, sepsis, bile leak, and pancreatitis ${ }^{(23)}$. The higher frequency of wound infection in the latter study is explained by their larger sample size (1005), compared to ours (56). In a study of equivalent sam- ple size, Koc et al. ${ }^{(24)}$ found that ERCP with LC was associated with a higher incidence of complications, including duodenal perforation (1.8\%), pancreatitis (3.7\%), and retained stones (5.5). Kadry et al. confirmed similar findings; the complication rate of ERCP was about $10 \%$ and about $50 \%$ of these complications were of major clinical importance like cholangitis, pancreatitis, CBD perforation, and hemorrhage $\mathrm{e}^{(25)}$. IOC reduced the morbidity rate to the lowest level in comparison to other modalities. In our study, the morbidity rate was $12.5 \%$, which seems higher in comparison to another Egyptian study where 49 patients underwent selective $\mathrm{IOC}$ during $\mathrm{LC}$ and had a morbidity rate of $8 \%(25)$. This is one of the drawbacks of using the morbidity rate, which weighs all complications evenly. A more efficient measure to assess morbidity is the postoperative morbidity 
index (PMI), which gives a score to each complication based on its severity. Hence, a $12.5 \%$ morbidity rate in our study would instead equals 0.038 on the scale of PMI.

\begin{tabular}{|l|c|c|}
\hline \multicolumn{3}{|c|}{ Table 4: Postoperative complications } \\
\hline Variables & $\mathrm{N}$ & $\%$ \\
\hline Intra-abdominal collection & 1 & 2 \\
\hline Wound infection & 2 & 3 \\
\hline Pancreatitis & 1 & 2 \\
\hline Bile leak & 1 & 2 \\
\hline Bleeding & 1 & 2 \\
\hline Postop. Hernia & 1 & 2 \\
\hline Bile duct injury & 0 & 0 \\
\hline Death & 0 & 0 \\
\hline
\end{tabular}

After one month of follow-up, few patients complained of abdominal pain (5\%), cholangitis (2\%), abnormal LFTs (5\%), and only $5 \%$ of patients were readmitted to hospital. By the second month, only $2 \%$ of patients had cholangitis and $2 \%$ had abnormal LFTs. After the third month, none of the patients had abdominal pain, jaundice, pancreatitis, cholangitis, abnormal LFTs, or was readmitted. A recent study showed that patients who had IOC had no readmissions after the surgery ${ }^{(26)}$. Khan et al.(19) compared between those who had $I O C$ and those who had not, and found that readmission rate was much higher in the latter group (0\% vs. $4 \%$ ) attributing readmission to abdominal pain and symptoms suggestive of CBD obstruction. The hospital length of stay was not prolonged except in $9 \%$ of cases for a maximum of four days. Most cases stayed at hospital for an average of 48 hours. This is in agreement with Mir et al.(27). Where they found that the mean hospital stay ranged between 18 and 72 hours. Similarly, Pham et al. 2016 reported a one to two days range for postoperative length of stay with the use of IOC during $\mathrm{LC}^{(28)}$. With IOC, we observed a conversion rate of $5 \%(\mathrm{~N}=3)$ to open cholecystectomy. This is smaller than the conversion rate reported by Horwood et al. at $7 \%(29)$. Alkhaffaf et al. showed a $7.2 \%$ conversion rate with IOC, compared to a $2.2 \%$ conversion rate with- out $10 C^{(30)}$. The reasons for conversion in most cases were adhesions, as well as poor anatomy delineation. Here comes the value of the surgeon's experience. In a previous study, the conversion rate with junior surgeons was higher than with experts ( $9 \%$ vs. $1.5 \%$ ); however this difference was not statistically significant(31). Concerning the IOC associated mortality rate, we observed a $0 \%$ mortality rate during the study period. Similarly a study was conducted in Kashmir where $0 \%$ mortality rate was observed as well ${ }^{(27)}$. This rate is low compared to ERCP-related mortality rate, which is approximately $0.4 \%$ (greater than that of LC itself)(32). Moreover, Tornqvist et al. claimed that early recognition of $\mathrm{BDI}$ using $\mathrm{IOC}$ resulted in a $62 \%$ reduction in mortality rate ${ }^{(20)}$. Most patients had no intervention (82\%). Two patients had failed CBD exploration and were referred for postoperative ERCP, one of them had large CBD stone difficult to extract with dormia basket and the other had dilated CBD without stones. After $I O C$, there was a sharp cut-off distally. post-operative ERCP revealed cholangiocarcinoma of distal CBD. Another two cases were managed conservatively by irrigation, flushing of CBD by saline and antispasmodics. Three cases were managed by flushing and milking from cystic duct-CBD junction, while another three cases were converted to open cholecys- 
tectomy. None of the patients had CBD

exploration, either trans-cystic or direct.

\begin{tabular}{|l|c|c|}
\hline \multicolumn{3}{|c|}{ Table 5: Follow up details in 1,2 and 3 months } \\
\hline Variables & $\mathrm{N}$ & $\%$ \\
\hline 1-month Follow up & & \\
\hline Abdominal pain & 3 & 5 \\
\hline Jaundice & 0 & 0 \\
\hline Pancreatitis & 0 & 0 \\
\hline Cholangitis & 1 & 2 \\
\hline Abnormal LFTs & 3 & 5 \\
\hline Readmission & 3 & 5 \\
\hline 2-month Follow up & & \\
\hline Abdominal pain & 0 & 0 \\
\hline Jaundice & 0 & 0 \\
\hline Pancreatitis & 0 & 0 \\
\hline Cholangitis & 1 & 2 \\
\hline Abnormal LFTs & 1 & 2 \\
\hline Readmission & 0 & 0 \\
\hline 3-month Follow up & & \\
\hline Abdominal pain & 0 & 0 \\
\hline Jaundice & 0 & 0 \\
\hline Pancreatitis & 0 & 0 \\
\hline Cholangitis & 0 & 0 \\
\hline Abnormal LFTs & 0 & 0 \\
\hline Readmission & 0 & 0 \\
\hline LFT: liver function tests & & \\
\hline
\end{tabular}

\section{Conclusion}

IOC during LC was associated with a low morbidity rate and no mortalities. This confirms the effectiveness of $I O C$ in reducing the postoperative complications of LC.

\section{Conflict of Interest}

All authors confirm no financial or personal relationship with a third party whose interests could be positively or negatively influenced by the article's content.

\section{Funding Source}

None (authors confirm they did not receive any funding to do this work)

\section{References}

1. Giger $U$, Michel JM, Vonlanthen R, Becker K, Kocher $\mathrm{T}$, Krähenbühl $\mathrm{L}$. Laparoscopic cholecystectomy in acute cholecystitis: Indication, technique, risk and outcome. Langenbeck's Arch Surg. 2005;390: 373-80.

2. Dubois F, Icard P, Berthelot $G$, Levard H. Coelioscopic Cholecystectomy. Ann Surg. 1990;211:60-2.

3. Flowers JL, Zucker KA, Graham SM, Scovill WA I AL, RW B. Laparoscopic cholangiography: results and indica tions. Ann Surg. 1992;215:209-16.

4. Gupta V, Jain G. Safe laparoscopic cholecystectomy: Adoption of universal culture of safety in cholecystectomy. World J Gastrointest Surg. 2019;11:62-84.

5. Martin B, Ong EGP. Selective intraoperative cholangiography during laparoscopic cholecystectomy in children is justified. J Pediatr Surg. Elsevier Inc.; 2018;53:270-3.

6. Giulea C, Enciu O, Bîrcă T, Miron A. Selective intraoperative cholangio- 
graphy in laparoscopic cholecystectomy. Chir. 2016;111:26-32.

7. Flum DR. Intraoperative Cholangiography and Risk of Common Bile Duct Injury During Cholecystectomy. JAMA. 2003;289:1639.

8. Urbach DR. Rate of elective cholecystectomy and the incidence of severe gallstone disease. Can Med Assoc J. 2005;172:1015-9.

9. Pesce A, Piccolo G, La Greca G, Puleo S. Utility of fluorescent cholangiography during laparoscopic cholecystectomy: A systematic review. World J Gastroenterol. 2015;21:7877-83.

10. Ding GQ, Cai W, Qin MF. Is intraoperative cholangiography necessary during laparoscopic cholecystectomy for cholelithiasis? World J Gastroenterol. 2015;21:2147-51.

11. Polat FR, Abci I, Coskun I, Uranues S. The importance of intraoperative cholangiography during laparoscopic cholecystectomy. JSLS J Soc Laparoendosc Surg. 4:103-7.

12. Halawani HM, Tamim $H$, Khalifeh $F$, Mailhac A, Jamali FR. Impact of intraoperative cholangiography on postoperative morbidity and readmission: analysis of the NSQIP database. Surg Endosc. 2016;30:5395403.

13. Zroback C, Chow G, Meneghetti A, et al. Fluorescent cholangiography in laparoscopic cholecystectomy: The initial Canadian experience. Am J Surg. 2016;211:933-7.

14. Rizzuto A, Fabozzi M, Settembre A, et al. Intraoperative cholangiography during cholecystectomy in sequential treatment of cholecystocholedocholithiasis: To be, or not to be, that is the question A cohort study. Int J Surg. IJS Publishing Group Ltd; 2018;53:53-8.

15. Elm $E$ Von, Altman DG, Egger $M$, et al. The Strengthening the Reporting of Observational Studies in Epidemiology (STROBE) Statement: Guidelines for reporting observational studies *. Int J Surg. Elsevier Ltd; 2014;12:1495-9.
16. Berrevoet $F$, Biglari $M$, Sinove $Y$, De Baardemaeker L, Troisi R, de Hemptinne B. Outpatient laparoscopic cholecystectomy in Belgium: what are we waiting for? Acta Chir Belg. Taylor \& Francis; 2006;106:537-40.

17. Patel SC, Bhatt R. Laparoscopic cholecystectomy at the aga khan hospital, Nairobi. East Afr Med J. Kenya Medical Association; 2000;77.

18. Wherry DC, Marohn MR, Malanoski MP, Hetz SP, Rich NM. An external audit of laparoscopic cholecystectomy in the steady state performed in medical treatment facilities of the Department of Defense. Ann Surg. Lippincott, Williams, and Wilkins; 1996;224:145.

19. Khan OA, Balaji S, Branagan G, Bennett DH, Davies N. Randomized clinical trial of routine on-table cholangiography during laparoscopic cholecystectomy. $\mathrm{Br} J$ Surg. Wiley Online Library; 2011;98:362-7.

20. Törnqvist B, Strömberg C, Persson G, Nilsson $M$. Effect of intended intraoperative cholangiography and early detection of bile duct injury on survival after cholecystectomy: population based cohort study. Bmj. British Medical Journal Publishing Group; 2012;345:e6457.

21. Sheffield KM, Riall TS, Han Y, Kuo Y-F, Townsend CM, Goodwin JS. Association between cholecystectomy with vs without intraoperative cholangiography and risk of common duct injury. Jama. American Medical Association; 2013;310:812-20.

22. Buddingh KT, Nieuwenhuijs VB. The critical view of safety and routine intraoperative cholangiography complement each other as safety measures during cholecystectomy. J Gastrointest Surg. Springer; 2011;15: 1069-70.

23. Photi ES, El-Hadi A, Brown S, et al. The routine use of cholangiography for laparoscopic cholecystectomy in the modern era. JSLS J Soc Laparoendosc Surg. Society of Laparoendoscopic 
Surgeons; 2017;21.

24. Koc B, Karahan S, Adas G, Tutal F, Guven H, Ozsoy A. Comparison of laparoscopic common bile duct exploration and endoscopic retrograde cholangiopancreatography plus laparoscopic cholecystectomy for choledocholithiasis: a prospective randomized study. Am J Surg. Elsevier; 2013;206:457-63.

25. Kadry I, Reda M, Ahmed H, ZaaZou M. Safety and outcome of selective intraoperative Cholangiography in patients with Suspected duct stones. 2007;

26. Verma S, Wichmann MW, Gunning T, Beukes E, Maddern G. Intraoperative cholangiogram during laparoscopic cholecystectomy: A clinical trial in rural setting. Aust J Rural Health. Wiley Online Library; 2016;24:415-21.

27. Mir IS, Mohsin M, Kirmani O, et al. Is intra-operative cholangiography necessary during laparoscopic cholecystectomy? A multicentre rural experience from a developing world country. World J Gastroenterol WJG. Baishideng Publishing Group Inc; 2007;13:4493.

28. Pham X-BD, de Virgilio C, Al-Khouja L, et al. Routine intraoperative cholangiography is unnecessary in patients with mild gallstone pancreatitis and normalizing bilirubin levels. Am J Surg. Elsevier; 2016;212:1047-53.

29. Horwood J, Akbar F, Davis K, Morgan R. Prospective evaluation of a selective approach to cholangiography for suspected common bile duct stones. Ann R Coll Surg Engl. The Royal College of Surgeons of England; 2010;92:206-10.

30. Alkhaffaf B, Parkin E, Flook D. Endoscopic retrograde cholangiopancreatography prior to laparoscopic cholecystectomy: a common and potentially hazardous technique that can be avoided. Arch Surg. American Medical Association; 2011;146:329-33.

31. Herrero A, Philippe C, Guillon F, Millat $B$, Borie $F$. Does the surgeon's experience influence the outcome of laparoscopic treatment of common bile duct stones? Surg Endosc. Springer; 2013;27:176-80.

32. Sirinek KR, Schwesinger $\mathrm{WH}$. Has intraoperative cholangiography during laparoscopic cholecystectomy become obsolete in the era of preoperative endoscopic retrograde and magnetic resonance cholangiopancreatography? J Am Coll Surg. Elsevier; 2015;220:522-8. 